\title{
Criando Falsas Memórias em Adultos por meio de Palavras Associadas
}

\author{
Lilian Milnitsky Stein ${ }^{1}$ \\ Giovanni Kuckartz Pergher \\ Pontifícia Universidade Católica do Rio Grande do Sul
}

\begin{abstract}
Resumo
Utilizou-se o procedimento de palavras associadas (DRM), o qual consiste na apresentação de listas de palavras semanticamente associadas às palavras não apresentadas, seguido por um teste de memória. Investigou-se o efeito do momento da testagem nas memórias verdadeiras e falsas e sua persistência por um período de uma semana, bem como o efeito de uma mera testagem sobre as respostas de um teste posterior. No Experimento 1, os índices de falsos reconhecimentos equivaleram aos dos verdadeiros em ambos os testes, sendo que as respostas verdadeiras persistiram mais que as falsas. No Experimento 2, no teste imediato, o índice de respostas verdadeiras foi superior ao das falsas, relação essa invertida no teste posterior. Quanto ao efeito da mera testagem, observou-se uma preservação das memórias verdadeiras e uma criação de falsas memórias. Os resultados são discutidos à luz da Teoria do Traço Difuso e consideradas possíveis implicações na prática psicoterápica e forense.

Palavras-chave: Falsas memórias; palavras associadas; teoria do traço difuso; psicologia cognitiva.
\end{abstract}

\section{Creating False Memories in Adults using Word Associated Lists}

\begin{abstract}
We used the DRM paradigm, which consists of the presentation of lists of associated words to a nonpresented one. We investigated the effect of time of testing, memory persistent and a mere memory test on both true and false memories. In Experiment 1, false recognition rates were as high as true recognition both in the immediate and delayed test. True memories were more persistent than false memories. Experiment 2 produced higher true recognition rates than false recognition in the immediate test. That relation was the inverse one in the delayed test. Regarding the effect of a previous memory test on a delayed one, we observed both a true memory inoculation against forgetting and a false memory creation effect. In addition, false memories were as persistent as true memories. Findings were discussed in terms of fuzzy trace theory interpretations. Possible clinical and forensic implications of our results were also considered.

Keywords: False memories; word associated; fuzzy trace theory; cognitive psychology.
\end{abstract}

Ao longo deste século, os pesquisadores têm se interessado pelos estudos da falsificação da memória, o fato de lembrarmos de eventos que na realidade não ocorreram, e de como se dá esse processo. As falsas memórias referem-se a uma gama de fenômenos que têm sido observados tanto em pesquisas experimentais, quanto no âmbito da psicoterapia, na área jurídica, bem como em outras variadas situações do cotidiano. As questões relacionadas à habilidade de crianças e adultos em relatar fidedignamente os fatos vividos, tanto como vítimas de abuso físico ou sexual, quanto como testemunhas oculares de contravenções em geral, têm

\footnotetext{
${ }^{1}$ Endereço para correspondência: Pós-Graduação em Psicologia, Faculdade de Psicologia, PUCRS, Av. Ipiranga, 6681, Prédio 11, sala 933 Porto Alegre, RS, 90619-900. Fone: (51) 3320-3633, Ramal: 225. E-mail: lilian@pucrs.br
}

influenciado e incentivado os estudos científicos na área das falsas memórias. Em linhas gerais, as falsas memórias referem-se ao fato de lembrarmos de eventos que na realidade não ocorreram. Informações são armazenadas na memória e posteriormente recordadas como se tivessem sido verdadeiramente vivenciadas (Roediger \& McDermott, 2000).

Roediger (1996) aponta que os primeiros experimentos demonstrando a ilusão ou falsificação da memória em crianças foram de Binet em 1900, na França, e de Stern em 1910, na Alemanha. O primeiro autor a pesquisar a falsificação da memória em adultos foi Bartlett (1932). Bartlett descreve o recordar como sendo um processo reconstrutivo, baseado em esquemas e conhecimento geral prévio do participante, salientando o papel da compreensão nas suas lembranças. Bem mais tarde, Loftus e Palmer (1974) introduziram um novo procedimento para o estudo das falsas memórias em 
adultos, chamado de procedimento de sugestão de falsa informação, onde, logo após a experiência vivida, é apresentada uma informação falsa compatível com esta experiência. Os resultados da aplicação deste procedimento têm produzido o chamado efeito de falsa informação (misinformation effect, Loftus \& Hoffman, 1989), qual seja, uma diminuição dos índices de reconhecimentos verdadeiros e um aumento significativo dos falsos.

Algumas falsas memórias são geradas espontaneamente, como resultado do processo normal de compreensão, ou seja, fruto de processos de distorções mnemônicas endógenas. Estas são as chamadas falsas memórias espontâneas ou autosugeridas (Brainerd \& Reyna, 1995). Por exemplo, você pode lembrar vividamente que ouviu sua colega dizer que a prova de biologia seria no dia dois de maio. Na verdade, as exatas palavras da colega teriam sido "Vamos ter uma prova de biologia logo após um feriado". É claro que o dia dois de maio é logo após um feriado, porém existe uma grande diferença entre lembrar-se exatamente do que você ouviu da colega e relatar uma inferência consistente com o que você ouviu. Esta distinção é fundamental, especialmente em situações onde se espera que a pessoa relate suas experiências de forma fidedigna e não suas inferências ou entendimento sobre o que ocorreu, como no caso de um testemunho num tribunal (Ceci \& Bruck, 1995; Reyna, 1998).

Outro tipo de falsas memórias pode resultar de sugestão externa, acidental ou deliberada, de uma informação falsa (Reyna, 1995), a qual não fez parte da experiência vivida pela pessoa, mas que de alguma forma é compatível com a mesma como no procedimento de sugestão de falsa informação. Assim, se a pessoa viu um carro que não parou numa placa de "dê a preferência" e, posteriormente, lhe é sugerido que a placa de trânsito era de "pare", poderá lembrar do sinal de pare como tendo sido realmente visto por ela (Loftus, Miller \& Burns, 1978). A pessoa passa a recordar de fatos como se tivessem sido realmente vividos, quando, na verdade, estes fatos foram-lhe sugeridos. As chamadas falsas memórias implantadas ou sugeridas (Loftus, 1979) podem resultar deste tipo de sugestão externa.

O fenômeno das falsas memórias tem sido mais recentemente estudado com base na Teoria do Traço Difuso (Fuz:y -Trace Theory, Brainerd \& Reyna, 1998; Reyna \& Brainerd, 1998; Reyna \& Lloyd, 1997), que identifica dois tipos de memórias: a de essência e a literal. A memória de essência é ampla, robusta e armazena somente as informações inespecíficas, ou seja, aquelas que representam o significado da experiência como um todo. Já a memória literal seria a codificação das informações de forma precisa, de modo que os detalhes são registrados e armazenados de forma episódica, sendo, contudo, mais suscetível ao esquecimento e à interferência, se comparada à memória de essência.

A presente pesquisa realizou os primeiros estudos brasileiros na área de falsas memórias utilizando um procedimento experimental que tem sido extensivamente empregado no estudo das falsas memórias em adultos (ver Roediger \& McDermott, 2000, e Roediger, McDermott \& Robison, 1998, para revisão), o chamado procedimento de Deese/Roedieger/McDermott (DRM) de listas de palavras associadas (Roediger \& McDermott, 1995). O procedimento das palavras associadas, que foi adaptado de um antigo estudo de Deese (1959), faz uso de várias listas de palavras. Cada lista, contendo palavras denominadas alvos, gira em torno de um mesmo tema. Por exemplo, todas as palavras da lista cama, descanso, acordar, cansado, sonho, despertador, soneca, cobertor, cochilo, sono, ronco, paz, bocejar e sonolento versam sobre o tema dormir. O procedimento experimental consiste primeiramente em uma etapa de apresentação desses conjuntos de listas, de forma aleatória, para serem memorizados. Em uma segunda etapa, a do teste de memória de reconhecimento, é apresentada uma nova lista de palavras composta por algumas palavras alvo, acrescida de palavras distratoras. Estas últimas podem ser de dois tipos: distrator crítico e distrator não relacionado. O distrator crítico é uma palavra que traduz a essência temática de cada uma das listas de palavras alvo, estando, por conseguinte, semanticamente associada a todas as palavras de uma mesma lista. No exemplo da lista acima, a palavra dormir seria um distrator crítico. Já o distrator não relacionado é uma palavra que não apresenta relação semântica com nenhuma palavra da lista. $\mathrm{O}$ teste de reconhecimento pode ser imediato (logo após a etapa de memorização do conjunto de listas) e/ou posterior (um determinado período de tempo após a etapa de memorização, que pode variar de horas, dias ou semanas). A tarefa do participante no teste de memória é reconhecer somente as palavras alvo e rejeitar qualquer outra palavra que não o seja, neste caso, tanto os distratores críticos quanto os não relacionados.

As 24 listas de palavras associadas de Roediger e McDermott (1995), juntamente com outras 12 listas de McDermott (1996b) passaram por um processo de normatização (Stadler, Roediger \& McDermott, 1999). Neste processo, foram verificadas as listas, com maior e menor capacidade, de produzirem falsas memórias, tanto em testes de recordação como de reconhecimento. Tendo em vista que a questão de interesse no procedimento de palavras associadas reside na associação semântica entre 
as palavras de uma mesma lista (alvos) e o distrator crítico, foi realizado um controle de freqüência de ocorrência de associação entre as palavras baseado nas normas de Russell e Jerkins (1954).

$\mathrm{Na}$ pesquisa original realizada por Roediger e McDermott (1995), os participantes, em um primeiro experimento, estudaram seis listas de doze palavras cada (denominadas alvos), sendo que as palavras de cada lista estavam semanticamente associadas com uma palavra não apresentada - denominada distrator crítico, a qual traduzia a essência semântica da lista. Em um teste de recordação realizado logo após o estudo das listas, os participantes evocavam os distratores críticos em $40 \%$ das tentativas. O teste de recordação foi seguido por um teste de reconhecimento, sendo constatado que não houve diferenças entre a probabilidade para os reconhecimentos verdadeiros $(86 \%)$ e para os reconhecimentos falsos dos distratores críticos $(84 \%)$. Os resultados do primeiro experimento estimularam a realização de um segundo, constituído por 24 listas de 15 palavras cada. Neste Experimento 2, o teste de recordação revelou uma taxa de intrusão de palavras não apresentadas de $55 \%$. O teste de reconhecimento, por sua vez, revelou índices mais altos de lembrança das palavras críticas não apresentadas ( $76 \%$ ) em relação às apresentadas $(72 \%)$.

Recentes estudos (Anastasi, Rhodes \& Burns, 2000; Brainerd \& Reyna, 1998b; Payne, Elie, Blackwell \& Neuschatz, 1996; Robinson \& Roediger, 1997; Schacter, Verfaellie \& Pradere, 1996), utilizando o procedimento de palavras associadas, constataram altos níveis de falsas memórias, mesmo imediatamente após os eventos terem sido apresentados, sem que tenha havido a interferência de informações falsas sugeridas.

A Teoria do Traço Difuso (Reyna \& Lloyd, 1997) explica estes resultados, fundamentada no princípio de que, no teste de memória, os itens distratores críticos e os alvos acessam diferentes tipos de memórias, que foram armazenadas durante a fase inicial de apresentação das listas (fase de memorização). A apresentação dos alvos na fase de memorização leva ao armazenamento de dois tipos dissociados de memórias. Uma delas armazena a forma superficial dos alvos (memória literal) e outra retém os vários significados desses alvos (memória da essência) (Brainerd, Stein \& Reyna, 1998). As palavras das listas DRM ativam temáticas comuns para adultos (p. ex.: móveis, vestuários, insetos, etc.), produzindo memórias da essência robustas acerca destas temáticas. Provavelmente porque as memórias de essência são tão fortes, elas predominem no processamento do teste de memória (Brainerd, Reyna \& Forrest, 2001). Num teste de memória, o correto reconhecimento dos itens-alvo pode ser baseado tanto na recuperação de traços literais ou da essência, ainda que em um teste imediato as memórias literais provavelmente predominem (Reyna \& Kiernan, 1994). Já o falso reconhecimento de distratores (como por exemplo, dormir), conforme Brainerd, Reyna e Kneer (1995), provavelmente estará baseado em memórias da essência, uma vez que a recuperação de memórias literais leva à correta rejeição desses distratores (p. ex., eu lembro das palavras cama, acordar, etc., e não havia na lista a palavra dormir). No caso específico do procedimento das palavras associadas, um distrator crítico como dormir é uma pista eficaz para a recuperação da memória da essência, já que representa o tema central, ao qual todas as palavras de cada lista estão semanticamente associadas.

A Teoria do Traço Difuso postula que, principalmente num teste de memória inicial, os itens-alvo (respostas verdadeiras) levam predominantemente à recuperação de memórias literais, e que os distratores críticos (alarmes falsos) levam à recuperação de memórias da essência. De acordo com um já conhecido pressuposto sobre a memória (Kintsch, Welsch, Schmalhofer \& Zimny, 1990), com a passagem do tempo, as memórias literais tornamse inacessíveis mais rapidamente que as da essência. Assim, com o tempo, a base mnemônica para as respostas verdadeiras tende a falhar mais rapidamente que aquela dos alarmes falsos.

Uma outra questão, que possui importância tanto teórica quanto aplicada, é se a administração de um mero teste de memória, cujo único objetivo é diagnosticar os conteúdos da memória, pode interferir nesses mesmos conteúdos. Brainerd e Ornstein (1991) demonstraram claramente que um teste de memória pode servir como uma proteção contra o esquecimento quando a memória for testada um tempo depois. Porém, Brainerd e Reyna (1996), como também Stein (1998), demonstraram que, além desse efeito de preservação da memória, um mero teste inicial pode, também, provocar um aumento das falsas memórias num teste posterior. Todavia, o possível efeito diferencial de um mero teste nas memórias verdadeiras e falsas ainda não foi testado dentro do contexto do procedimento das palavras associadas.

Com base nos pressupostos teóricos acima delineados, levantou-se quatro hipóteses que foram testadas nos dois experimentos que realizamos:

a) $\mathrm{O}$ índice de reconhecimento verdadeiro pode ser semelhante ao de reconhecimento falso num teste de memória;

b) $\mathrm{O}$ índice de reconhecimento falso será mais alto que o de reconhecimento verdadeiro no teste de memória posterior quando comparado ao teste de memória imediato; 
c) Os reconhecimentos falsos persistirão mais ao longo de uma semana do que os reconhecimentos verdadeiros;

d) Um teste de memória anterior provocará um efeito de magnitude semelhante tanto nos índices de reconhecimento verdadeiros quanto falsos.

Com o objetivo de testar estas quatro hipóteses, desenvolvemos dois experimentos utilizando uma adaptação do procedimento das palavras associadas.

\section{Experimento 1}

No primeiro experimento, buscamos obter dados preliminares em adultos utilizando este procedimento, no sentido de verificar o efeito do momento da testagem (imediato e posterior) na capacidade dos participantes de conseguirem diferenciar os alvos (memórias verdadeiras), dos distratores críticos (memórias falsas) e dos distratores não relacionados (respostas de viés). Além disso, buscamos verificar se haviam diferenças na durabilidade das memórias verdadeiras e falsas, ou seja, se haviam diferenças na persistência dessas memórias por um período de uma semana.

\section{Método}

\section{Participantes}

Participaram 27 alunos de graduação em Psicologia de uma universidade particular de Porto Alegre, sendo 26 do sexo feminino e 1 do masculino, com idade média de 22,26 anos $(d p=5,08)$. A maioria dos participantes pertencia a um nível sócio-econômico médio/médio alto. Todos eram alunos de uma mesma turma e foram escolhidos por conveniência, sendo sua participação voluntária.

\section{Instrumentos}

Traduzimos e adaptamos dez das 36 listas de palavras associadas de Stadler, Roediger e McDermott (1999). As listas de palavras originais em inglês foram independentemente traduzidas para o português por três juizes que dominavam plenamente os idiomas em questão. Feita a tradução, dois outros avaliadores, também com proficiência em inglês e português, construíram as listas de palavras originais definitivas, tendo por base os seguintes critérios: (a) quando as três traduções dos juizes concordavam, a palavra em questão era automaticamente incluída na lista original; (b) quando as traduções eram diferentes, os avaliadores buscavam um consenso e a palavra que melhor se adaptasse à realidade brasileira era selecionada.

Desta forma, cada uma das dez listas originais ficou composta por quinze palavras semanticamente associadas (p. ex.: mesa, sentar, pernas, assento, poltrona, escrivaninha, reclinável, sofá, madeira, estofado, giratória, banqueta, sentado, balançando, encosto), tendo uma palavra (distrator crítico) correspondente que representava o tema de cada uma das listas (no exemplo anterior: cadeira). O material original era composto pelas dez listas em ordem aleatória (Anexo A). A disposição das listas foi definida através de uma tabela de números aleatórios. Dentro de cada uma das listas, as palavras associadas foram apresentadas em ordem decrescente de associação semântica com o tema da lista, ou seja, as primeiras palavras de cada lista eram mais associadas semanticamente (i. e., compartilhavam mais o sentido) com o distrator crítico que as últimas palavras (Roediger \& McDermott, 1995). Portanto, o material original continha um total de 150 palavras. Os distratores críticos, de cada lista, não foram incluídos na lista original.

O teste de memória imediato foi constituído por 70 palavras, sendo 10 itens distratores críticos (palavras que representavam o tema de cada uma das listas e que não foram apresentadas no material original), 40 itens-alvo (palavras que foram apresentadas no material original) e 20 itens distratores não relacionados (palavras sem relação semântica com o material original). Todos os distratores críticos foram utilizados como itens do teste imediato. Os 40 itens-alvo foram escolhidos aleatoriamente (utilizando-se uma tabela de números aleatórios) entre as posições 3-12 (inclusive) de suas respectivas listas, sendo que somente quatro itens-alvo foram escolhidas de cada lista. Os 20 distratores não relacionados foram selecionados a partir de dez outras listas de palavras associadas de Stadler, Roediger e McDermott (1999), das quais selecionou-se duas palavras por lista. A ordem dos itens do teste imediato foi aleatória (utilizando-se o mesmo método descrito anteriormente), com a única restrição de que alvos e distratores críticos da mesma lista não aparecessem em seqüência imediata.

Como tarefa de distração (também denominada de tarefa de isolamento) foi utilizada uma atividade na qual os participantes deviam descobrir, em no máximo três minutos, qual o número que completava uma determinada seqüência de algarismos, segundo uma lógica a ser desvendada. O objetivo desta tarefa foi controlar os conhecidos efeitos de primazia e recência (Murdock, 1962), ou seja, o fato dos participantes evocarem com mais facilidade as primeiras e as últimas palavras de uma lista, através da apresentação desta nova tarefa para que a atenção dos participantes fosse direcionada para um material diferente da lista alvo. O tempo total de duração desta tarefa de isolamento foi de aproximadamente seis minutos. No teste de memória posterior, empregou-se 
exatamente a mesma lista de palavras do teste de memória imediato, com 70 palavras.

\section{Procedimentos}

Os participantes foram testados em grupo, em sua sala de aula. Eles foram instruídos a ouvirem com atenção a lista de palavras alvo, pois a memória deles para aquelas palavras iria ser testada posteriormente (Anexo B). A apresentação dos alvos foi feita através de gravação em áudio, com um intervalo de dois segundos entre cada uma das palavras. Após escutarem a lista alvo, os participantes realizaram a tarefa de distração.

Em seguida, os participantes receberam a folha de respostas e instruções detalhadas de como responder o teste de memória. As instruções também continham alguns exemplos ilustrativos com outras palavras, que não apresentavam nenhuma relação com a lista alvo. Os participantes foram instruídos a assinalar com um " $x$ " na resposta sim somente quando reconheciam a palavra apresentada no teste de memória como tendo sido escutada na lista alvo e, para assinalar com um " $\mathrm{x}$ " no não, se não haviam escutado-a anteriormente. Assim sendo, apresentou-se a lista de 70 palavras do teste imediato. A lista foi lida em voz alta e pausadamente por um dos experimentadores, com um intervalo de dois segundos entre cada palavra. Optou-se pela apresentação oral da lista de palavras do teste de memória por um dos experimentadores, que não era o mesmo da gravação em áudio da lista alvo, com o objetivo de criar uma clara diferenciação entre a lista do teste e a alvo. Segundo a hipótese de especificidade de codificação de Tulving (1983), a palavra armazenada na memória também retém informações sobre o contexto em que estava inserida (no caso a voz e a forma de apresentação áudio-gravada). Terminado o teste imediato, explicou-se aos participantes que, em uma semana, uma nova tarefa seria realizada.

Seguindo o modelo de uma série de estudos na área de falsas memórias (Tsai, Loftus \& Polage, 2000), utilizamos uma semana como período de intervalo para a testagem posterior. Uma semana depois, voltamos à sala onde foi feita a primeira aplicação. Os participantes foram lembrados que iríamos testar a memória deles para aquela lista apresentada uma semana atrás na gravação de áudio. Em seguida, foram distribuídas as folhas de respostas e repetidas as mesmas instruções do teste imediato. Os procedimentos de aplicação do teste de memória posterior foram idênticos aos do teste imediato.

Neste experimento, portanto, duas variáveis independentes foram manipuladas intra-participantes. A primeira delas, tipo de resposta no teste de memória, possuía três níveis: verdadeira (reconhecimento de itens

Psicologia: Reflexão e Crítica, 2001, 14(2), pp. 353-366 alvo), falsa (reconhecimento de itens distratores críticos) e de viés (reconhecimento de itens distratores não relacionados). A segunda variável foi o momento da testagem, com dois níveis: imediato (teste de memória realizado imediatamente após a apresentação do material original) e posterior (teste de memória realizado uma semana após). Assim sendo, utilizamos um delineamento experimental 3 (tipo de resposta: verdadeira, falsa, de viés) $\mathrm{X} 2$ (momento da testagem: imediato, posterior) no Experimento 1.

Como variável dependente foi utilizada a performance no teste de memória, obtida através da média de reconhecimento para os itens do teste. Assim, cada vez que o participante aceitava um determinado item, era computado o valor $1 \mathrm{e}$, cada vez o participante rejeitava um determinado item, era computado o valor 0 .

Os dados de ambos testes de memória foram testados através de análises de variância multivariada para medidas repetidas (ANOVA). Os efeitos evidenciados pelas ANOVAs foram posteriormente verificados através de testes $t$. Também foram utilizadas análises de correlação bivariada de Pearson. Foi fixado um nível de significância de $p<0,05$ para todo o tratamento estatístico empregado.

\section{Resultados}

Os resultados serão apresentados fazendo-se referência a três tipos de respostas nos testes de memória. A primeira delas, resposta verdadeira, diz respeito à média de reconhecimento (responder "sim") aos itens-alvo, apresentados no material original. É este tipo de resposta que vai fornecer o índice de memórias verdadeiras. $\mathrm{O}$ segundo tipo de resposta, falsa, refere-se à média de aceitação dos itens distratores críticos (palavras não apresentadas no material original e que resumem a essência semântica das listas). A taxa de falsas memórias será baseada neste último tipo de resposta. Por fim, temos as respostas de viés, as quais são computadas através das médias de aceitação dos distratores não relacionados (palavras sem associação semântica com o material original). Os índices de respostas de viés fornecem a medida de respostas não mnemônicas dos participantes.

Os resultados, neste primeiro experimento, evidenciaram que a adaptação do procedimento de palavras associadas produziu efeitos similares àqueles obtidos na literatura original em inglês (McDermott \& Roediger, 1998; Roediger \& McDermott, 1995). A ANOVA que testou o efeito das variáveis momento da testagem e tipo de resposta produziu efeitos principais tanto para a primeira, $F(1,26)=5,985, p<0,05$, quanto para a segunda $F(2,25)=146,102, p<0,001$. Estes efeitos 
principais foram qualificados por uma interação entre o efeito do momento da testagem por tipo de resposta $F(2,25)=25,521, p<0,001$. Testes adicionais evidenciaram que as diferenças entre as médias de respostas de viés em relação às médias de respostas verdadeiras e falsas é que foram responsáveis por esta interação, $t s(26)>9,653$, ps $<0,01$. Conforme a Tabela 1 , as médias dos índices de respostas falsas (falsas memórias) foram estatisticamente semelhantes aos índices de respostas verdadeiras (memórias verdadeiras), tanto no teste imediato, $t(26)=$ $0,413, p>0,05$, como no teste posterior $t(26)=0,356$, $p>0,05$.

Quanto à questão referente à durabilidade da memória, testamos o efeito de persistência das respostas ao longo eram colegas de uma mesma turma (diferente daquela do Experimento 1), sendo a participação voluntária. A escolha da amostra foi por conveniência. A maioria dos participantes pertencia a um nível sócio-econômico médio/médio alto.

\section{Instrumentos}

Todos os instrumentos foram idênticos ao do Experimento 1, com exceção do teste de memória imediato. Este teste foi baseado em somente cinco listas, selecionadas aleatoriamente dentre as dez que compunham o material original. $\mathrm{O}$ teste de memória imediato apresentava a seguinte composição: cinco distratores críticos (correspondentes às cinco listas

Tabela 1. Média do Índice de Reconhecimento por Tipo de Item e Momento da Testagem ( $d p$ ) no Experimento 1

\begin{tabular}{llll}
\hline \multirow{2}{*}{ Momento do teste } & Alvos & Dipo de Item \\
\cline { 2 - 4 } & \multicolumn{2}{l}{ Distratores Críticos } & Distratores não Relac. \\
\hline Imediato & $0,6657(0,1305)$ & $0,6778(0,1553)$ & $0,1037(0,1192)$ \\
Posterior & $0,6472(0,1382)$ & $0,6593(0,1866)$ & $0,2389(0,1571)$ \\
\hline
\end{tabular}

de uma semana através de análises de correlação bivariada de Pearson. Estas análises demonstraram que as respostas verdadeiras persistiram $(r=0,758, p<0,001)$ mais que as falsas $(r=0,512, p<0,01)$. Ainda que supostamente as respostas de viés não tenham uma base mnemônica sobre o material original, o resultado da análise da persistência dessas respostas ao longo de uma semana foi semelhante ao das respostas verdadeiras $(r=0,777, p<0,001)$. Passamos a apresentação do segundo experimento para então discutirmos os resultados de ambos estudos.

\section{Experimento 2}

No segundo experimento, além de investigar os efeitos do momento da testagem e da persistência da memória, buscamos também verificar o efeito de uma mera testagem de memória anterior sobre as respostas de um teste de memória posterior.

\section{Método}

\section{Participantes}

Participaram 33 alunos de graduação em Psicologia de uma universidade particular de Porto Alegre, sendo 32 do sexo feminino e 1 do masculino. A idade média dos participantes foi de 21,73 anos $(d p=4,96)$. Todos selecionadas), 20 alvos (somente oriundos das mesmas cinco listas) e dez distratores não relacionados, perfazendo um total de 35 itens. A escolha dos alvos e distratores não relacionados, bem como sua ordem de apresentação foi feita aleatoriamente, seguindo os mesmo critérios do Experimento 1. O teste de memória posterior foi idêntico ao do Experimento 1.

\section{Procedimentos}

Os participantes receberam as mesmas instruções dadas no Experimento 1, ou seja, ouviram a audiogravação da lista original, realizaram a atividade distratora e, em seguida, receberam o teste de memória imediato com 35 itens. Uma semana depois, foi aplicado o teste de memória posterior com 70 itens, seguindo os mesmos procedimentos do Experimento 1.

O Experimento 2 envolveu três variáveis independentes, todas manipuladas intra participantes. A primeira delas, tipo de resposta, possuía três níveis: verdadeira (reconhecimento de itens alvo), falsa (reconhecimento de itens distratores críticos) e de viés (reconhecimento de itens distratores não relacionados). A segunda variável foi o momento da testagem, com dois níveis: imediato (teste de memória realizado imediatamente após a apresentação do material original) e posterior (teste de memória realizado uma semana após). 
A terceira variável, mera testagem, também apresentava dois níveis: itens testados (itens do teste posterior que haviam sido testados no teste imediato) e itens não testados (itens do teste posterior que não haviam sido testados no teste imediato). Desta maneira, utilizamos um delineamento experimental 3 (tipo de resposta: verdadeira, falsa, de viés) X 2 (momento da testagem: imediato, posterior) X 2 (mera testagem: itens testados, itens não testados). A variável dependente, bem como seus procedimentos de cálculo, foi idêntica ao do Experimento 1.

Os dados de ambos testes de memória foram testados através de análises de variância multivariada para medidas repetidas (ANOVA). Os efeitos evidenciados pelas ANOVAs foram posteriormente verificados através de testes $t$. Também foram utilizadas análises de correlação parcial de Pearson. Foi fixado um nível de significância de $p<0,05$ para todo o tratamento estatístico empregado.

\section{Resultados}

Com o objetivo de controlar o efeito de um mero teste de memória anterior nas respostas do teste posterior, as análises a seguir foram realizadas comparando-se os índices de reconhecimento no teste imediato com os índices de reconhecimento no teste posterior, somente para os itens não testados anteriormente, isto é, os 35 itens do teste posterior que não foram apresentados no teste imediato. A ANOVA que testou o efeito das variáveis momento da testagem e tipo de resposta produziu efeitos principais tanto para o momento da testagem, $F(1,32)$ $=9,802, p<0,01$, quanto para o tipo de resposta, $F(2,31)$ $=62,260, p<0,001$. O índice geral de reconhecimento no teste imediato foi maior $(m=0,398, d p=0,106)$ que no teste posterior $(m=0,308, d p=0,152)$. Quanto às diferenças entre os tipos de resposta, replicando os resultados do Experimento 1, testes adicionais $(t s(32)>$ $7,675, p s<0,001)$ evidenciaram que somente o índice de respostas de viés $(m=0,152, d p=0,129)$ foi inferior ao das respostas verdadeiras $(m=0,457, d p=0,112)$ e ao das respostas falsas ( $m=0,452, d p=0,184)$, uma vez que não houve diferenças estatisticamente significativas entre esses dois últimos tipos de resposta $(t(32)=0,203, p>0,05)$.

Os efeitos principais de momento da testagem e tipo de resposta foram qualificados por uma interação, $F(2,31)$ $=39,473, p<0,001$, apresentada na Figura 1. Pode-se observar que, no teste imediato, o índice de respostas verdadeiras $(m=0,623, d p=0,157)$ foi superior ao de respostas falsas $(m=0,448, d p=0,229)$, sendo estas diferenças estatisticamente significativas, $t(37)=4,657$, $p<0,001$. Essa relação, entretanto, inverte-se no teste posterior. Uma semana depois, o índice médio de

Psicologia: Reflexão e Crítica, 2001, 14(2), pp. 353-366 respostas verdadeiras cai $(m=0,291, d p=0,166)$, mantendo-se o índice médio de respostas falsas $(m=0,455$, $d p=0,288 ; t(32)=-3,745, p<0,01)$. Não houve diferenças estatisticamente significativas no que concerne às respostas falsas nos testes imediato e posterior, $t(32)=-0,094$, $p>0,05)$. Com a passagem do tempo, também há um aumento das respostas de viés, evidenciado pelas diferenças no índice de aceitação dos distratores não relacionados nos testes imediato $(m=0,124, d p=0,13) \mathrm{e}$ posterior $(m=0,179, d p=0,155 ; t(32)=-2,52, p<0,05)$. Todavia, como pode constatar-se na Figura 1, os índices de respostas com base mnemônica, sejam verdadeiras ou falsas, foram muito superiores aos das respostas de viés, tanto no teste imediato quanto no posterior.

Foi testado, também, o efeito de um mero teste de memória, o qual diz respeito às diferenças encontradas

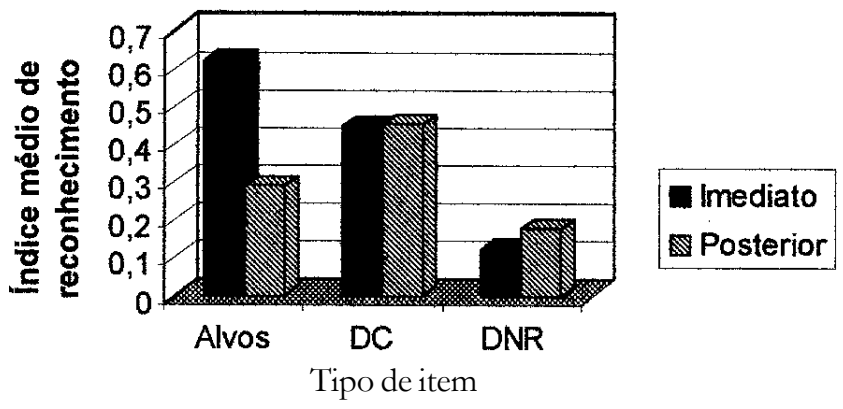

Figura 1. Média do índice de reconhecimento por tipo de item e momento da testagem no Experimento 2

no teste posterior em relação aos itens que foram ou não foram testados no teste imediato. Dois efeitos constituem o foco de interesse dessa análise: (1) o efeito de proteção das memórias verdadeiras contra o esquecimento, e (2) o efeito de criação de falsas memórias.

Os índices de reconhecimento no teste posterior foram analisados por uma ANOVA 2 (mera testagem: itens testados e não testados) X 3 (tipo de resposta: verdadeira, falsa e de viés) com medidas repetidas em ambas variáveis. Esta análise produziu um efeito principal para a mera testagem, $F(2,31)=164,509, p<0,001$, uma vez que o índice médio de reconhecimento no teste posterior dos itens anteriormente testados $(m=0,642, d p=$ $0,129)$ foi superior ao dos itens não testados $(m=0,308$, $d p=0,152)$. O efeito principal para o tipo de resposta, $F(2,31)=37,507, p<0,001$, evidenciou diferenças nas médias de reconhecimento no teste posterior para os distintos tipos de respostas. Como já visto na Figura 1, a relação dos índices de reconhecimento para esses tipos de respostas foi: falsas $>$ verdadeiras $>$ de viés. 
Contudo, o foco de interesse desta análise reside na interação entre as duas variáveis investigadas, quais sejam, mera testagem e tipo de resposta, $F(2,31)=18,005$, $p<0,001$. Como pode verificar-se na Figura 2, observouse um duplo efeito por mera testagem. Constatou-se um efeito de proteção das memórias verdadeiras contra o esquecimento, uma vez que o índice de respostas verdadeiras para os itens testados anteriormente $(m=0,774$, $d p=0,147)$ foi muito superior ao dos itens não testados $(m=0,291, d p=0,166)$. Observou-se também um efeito de criação de falsas memórias, já que o índice de respostas falsas para os itens testados anteriormente $(m=0,764$, $d p=0,215)$ foi muito superior ao dos itens não testados $(m=0,454, d p=0,288)$.

Como vimos, os resultados na Figura 2 indicam que um mero teste de memória anterior pode incrementar o índice de falsas memórias num teste posterior em níveis

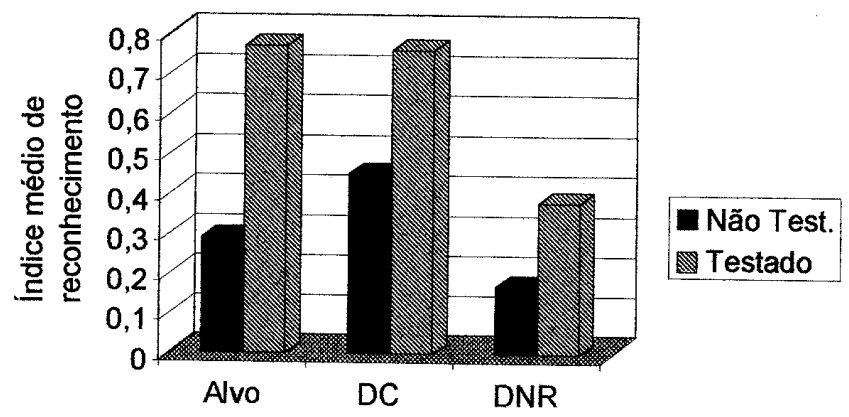

Figura 2. Média do índice de reconhecimento por tipo de item e mera testagem no Experimento 2

equivalentes ao das memórias verdadeiras. Portanto, a questão que se coloca é se um mero teste de memória produz uma melhora ou piora na precisão da memória num teste posterior, ou seja, se um mero teste aumenta ou diminui a acuracidade da memória num teste posterior. Para responder a esta questão, calculamos $d$, que é uma medida padronizada de detecção de sinal que permite avaliar a capacidade do participante de discernir as respostas verdadeiras das respostas falsas ${ }^{2}$. Encontramos que os valores de $d$ ' para os itens testados $\left(d^{\prime}=0,00494, d p=1,063\right)$ foi maior que para os itens não testados $\left(d^{\prime}=-0,567, d p=0,870\right)$, sendo esta diferença estatisticamente significativa, $t(32)=-2,240, p<0,05)$. Todavia, ainda que um mero teste de memória tenha produzido um pequeno ganho líquido na precisão da memória, este não foi suficientemente grande para

${ }^{2}$ A estatística $d^{\prime}=\left(\mathrm{p}_{\mathrm{v}}-\mathrm{p}_{\mathrm{f}}\right) / d p\left(\mathrm{p}_{\mathrm{f}}\right)$, onde $p_{v}$ é a probabilidade de respostas verdadeiras e $p_{f}$ a probabilidade de respostas falsas (McNicol, 1972). permitir que os participantes pudessem discernir as memórias verdadeiras das falsas, visto que o valor $d$ ' para os itens testados foi praticamente zero. Cabe ainda ressaltar que quando os itens não foram testados anteriormente, a memória dos participantes ficou bastante prejudicada, visto que o valor $d$ ' negativo indica que os participantes reconheceram melhor os distratores críticos que os próprios alvos.

Quanto à questão referente à durabilidade da memória, testamos o efeito de persistência das respostas ao longo de uma semana, através de análises de correlação parcial de Pearson, com controle para os itens não testados. Estas análises demonstraram que as respostas verdadeiras persistiram mais $(r=0,735, p<0,001)$ que as falsas $(r=0,572, p<0,01)$. As respostas de viés, pelo contrário, não persistiram $(r=0,313, p>0,05)$ ao longo de uma semana.

\section{Discussão}

Os dois experimentos aqui apresentados fornecem fortes evidências de que as falsas memórias podem ser induzidas utilizando-se um procedimento com listas de palavras associadas. Os principais resultados de nossos experimentos podem ser resumidos da seguinte forma: (1) quando foram apresentadas múltiplas listas de palavras associadas a palavras críticas (não incluídas nas listas), os participantes do estudo exibiram altos índices de falso reconhecimento para estes distratores críticos; e, (2) em contraste com o esquecimento normal observado para os itens estudados nas listas originais, não houve um decréscimo nos índices de falso reconhecimento após uma semana.

Os resultados encontrados estão de acordo com os evidenciados em outros estudos com listas de palavras associadas (por exemplo, Maylor \& Mo, 1999; Payne, Elie, Blackwell, \& Neuschatz, 1996; Roediger \& McDermott, 1995; Seamon, Luo, Schlegel, Greene \& Goldenberg, 2000; Zechmeister \& Zechmeister, 1996). Portanto, tanto a hipótese (a) de que os níveis de reconhecimento verdadeiros e os falsos poderiam assemelhar-se, quanto a hipótese (b) de que com a passagem do tempo os índices de falsas memórias poderiam superar aqueles das memórias verdadeiras foram corroboradas por nossos resultados. Estes achados estão em acordo como uma série de estudos recentes (Brainerd, Stein \& Reyna, 1998; Reyna \& Brainerd, 1995; Reyna \& Kiernan, 1994; Titcomb, 1996), em que a independência ou dissociação entre respostas para alvos e para distratores semanticamente relacionados ficou evidenciada. 
Nossos resultados podem ser explicados com base em pressupostos chaves da Teoria do Traço Difuso, quais sejam: a memória não é unitária, mas sim que representações dissociadas são armazenadas, variando tanto no seu grau de especificidade, desde traços literais e específicos até traços difusos que contém a essência da informação original, quanto no ritmo de desintegração destes traços com o passar do tempo (Reyna, 1998). Portanto, num teste de memória posterior, há uma perda mais significativa da base mnemônica (traços literais) para as memórias verdadeiras com relação àquela que alicerça as falsas memórias (traços da essência), elevando os índices de reconhecimento das últimas em relação às primeiras.

Ainda no Experimento 2, quando examinamos o efeito de um mero teste de memória anterior no desempenho de um teste de memória uma semana depois, observamos que, como previsto em nossa hipótese inicial (d), um teste de reconhecimento inicial contribuiu na prevenção contra o esquecimento das respostas verdadeiras (reconhecimento dos alvos num teste posterior). Nossos resultados estão em consonância com outros estudos como, por exemplo, os de Brainerd e Mojardin (1998), Brainerd e Reyna (1996) e Stein (1998), podendo-se postular que a preservação das memórias literais seria o fator responsável por esses achados.

Ainda em conformidade com a hipótese (d), um mero teste de memória também produziu um aumento nas respostas falsas (reconhecimento dos distratores críticos) no teste posterior, o chamado efeito de criação de falsas memórias (Brainerd \& Reyna, 1996). Este efeito pode ser fruto de dois processos mnemônicos distintos. Primeiro, os itens distratores do teste possibilitam a recuperação da memória da essência que foi armazenada no momento da apresentação da lista original (Brainerd, Reyna \& Forrest, 2001), memória esta que é compartilhada tanto pelo alvo quanto pelo distrator crítico (Brainerd \& Reyna, 1998a; Brainerd, Reyna \& Kneer, 1995). Segundo, o efeito de criação de falsas memórias pode, também, ser decorrente da recuperação da memória literal do distrator crítico apresentado no teste inicial. Assim, no teste de reconhecimento posterior, a apresentação do mesmo distrator crítico como item do teste pode levar à recuperação deste traço literal (Brainerd \& Reyna, 1996), fazendo com que a pessoa aceite o distrator crítico como palavra da lista original. Esta segunda situação vai ocorrer, sobretudo, se a informação sobre a fonte ou origem da memória literal for esquecida, ou seja, a pessoa não conseguir discernir se a memória literal recuperada foi armazenada durante a etapa da apresentação das listas originais ou do primeiro teste de memória (Johnson e cols., 1993).

Psicologia: Reflexão e Crítica, 2001, 14(2), pp. 353-366
Uma última questão se coloca frente aos resultados relativos ao efeito da mera testagem, a qual levantamos na hipótese (d): como a magnitude do efeito de prevenção das memórias verdadeiras foi semelhante daquela de criação de falsas memórias, qual o ganho na precisão da memória ao realizar-se um teste anterior? Segundo nossos resultados, muito pouco. Portanto, uma prática comum, tanto na área forense quanto clínica, que é submeter o indivíduo a múltiplas entrevistas com o único propósito de obter-se um relato mais fidedigno dos fatos em questão, pode ser falaciosa. Aliás, em determinadas situações, como aquelas de estudos anteriores tanto com lista de palavras de natureza distinta das utilizadas por nós (Brainerd \& Reyna, 1996; McDermott, 1996b), quanto em estudos que empregaram listas de frases (Brainerd \& Mojardin, 1998), o efeito de uma mera entrevista anterior pode levar a um prejuízo importante na precisão do relato em uma entrevista posterior, visto que o efeito de criação das falsas memórias pode suplantar aquele de proteção das memórias para o que realmente ocorreu (Reyna, 1998).

Com relação à persistência dos traços de memória por um período de uma semana, é senso comum que a memória para aquelas informações que fazem parte da experiência realmente vivida deve ser mais duradoura que para aquilo que não foi vivido. Todavia, contrariando nosso senso comum, as falsas memórias podem ser tão duradouras quanto às verdadeiras (McDermott, 1996a). Nossos resultados sobre a persistência da memória mostraram que, no caso de listas de palavras que compartilham uma mesma base semântica, ambas memórias verdadeiras e falsas foram duradouras, com uma certa superioridade das primeiras. Este último dado está em desacordo com nossa hipótese inicial (c), a qual postulava que os alarmes falsos persistirão mais ao longo de uma semana do que os reconhecimentos verdadeiros. Todavia, seguindo o mesmo pressuposto teórico da hipótese (c), a razão pela qual as memórias verdadeiras persistiram mais que as falsas em nossos dois experimentos, pode ter sido devido ao fato de uma mesma base semântica (memórias da essência) ser compartilhada por todas as palavras da mesma lista alvo. São justamente estas memórias da essência, as quais tem sido demonstrado serem mais duradouras que as literais (Brainerd, Reyna \& Brandse, 1995) que podem, também, ter servido de base para a aceitação dos itens alvos no teste imediato e posterior, além das memórias literais. Assim, ainda que as memórias literais sejam mais frágeis que as da essência, a superior persistência das respostas verdadeiras em relação as falsas pode dever-se ao fato de que as primeiras possuem dois substratos mnemônicos para embasar uma resposta correta. Já as respostas falsas 
possuem apenas um (i.e., as memórias da essência). Contudo, esta hipótese de que materiais como listas de palavras associadas geram uma possível solidez das memórias de essência, em função de seu compartilhamento por várias palavras de uma mesma lista, deve ser testada de forma mais específica. Neste sentido, recentes trabalhos Brainerd, Wright, Reyna e Mojardin (no prelo) e Brainerd, Reyna e Forrest (2001) mostraram que, no procedimento de palavras associadas, existe um fortalecimento das memórias da essência pela repetida ativação da mesma base semântica.

Finalmente, um último ponto pode ser levantado a partir de nossos resultados e que concerne diretamente questões aplicadas tanto à prática forense quanto a psicoterápica: falsos relatos podem ser bastante freqüentes em situações jurídicas ou clínicas que enfatizem a memória para a essência do que foi vivido. Em terapia, normalmente as sessões desenvolvem-se em torno de uma temática central (por exemplo, um trauma emocional ou físico), em que as experiências trazidas pelo paciente são exploradas em relação a este tema principal. O mesmo ocorre em situações jurídicas, nas quais tanto os procedimentos usuais de perícia psicológica quanto os questionamentos de testemunhas versam sobre um tópico central (neste caso, pode ser um crime que está sendo investigado). Portanto, tendo por base os resultados de nossos experimentos, não é de se admirar que seja bastante comum a situação em que pessoas, submetidas a psicoterapia ou sob investigação forense, produzam falsos relatos, que não sejam baseados em simulação (i.e., mentira), mas sim em memórias que substanciam o foco central do fato em questão.

\section{Referências}

Alba, J. W. \& Hasher, L. (1983). Is memory schematic? Psychological Bulletin, 93(2), 201-231.

Anastasi, J. S., Rhodes, M. G. \& Burns M. C. (2000). Distinguishing between memory illusions and actual memories using phenomenological measurements and explicit warnings. The American Journal of Psychology, 113,1-26.

Bartlett, F. C. (1932). Remembering: A study in experimental and social psychology. New York \& London: Cambridge University Press.

Binet, H. (1900). La suggestibilite. Paris: Schleicher Feres.

Brainerd, C. J. \& Mojardin, A. H. (1998). Children's spontaneous false memories for narrative statements: Long-term persistence and meretesting effects. Child Development, 69, 1361-1377.

Brainerd, C. J. \& Ornstein, P. A. (1991). Children's memory for witnessed events: The developmental backdrop. Em J. Doris (Org.), The suggestibility of children's recollections (pp. 10-20). Washington, D.C.: American Psychological Association.

Brainerd, C. J. \& Reyna, V. F. (1995). Autosuggestibility in memory development. Cognitive Psychology, 28, 65-101.

Brainerd, C. J. \& Reyna, V. F. (1996). Mere memory testing creates false memories in children. Developmental Psychology, 32, 467-478.
Brainerd, C. J., \& Reyna, V. F. (1998a). Fuzzy-trace theory and children's false memories. Journal of Experimental Child Psychology, 71, 81-129.

Brainerd, C. J., \& Reyna, V. F. (1998b). When things that were not experienced are easier to "remember" than things that were. Psychological Science, 9, 484-489.

Brainerd, C. J. Reyna, V. F. \& Brandse, E. (1995). Are children's false memories more persistent than their true memories? Psychological Science, 6,359-364.

Brainerd, C. J. Reyna, V. F. \& Forrest, T. J. (2001). Are young children more susceptible to the false-memory illusion? Manuscrito submetido para publicação. University of Arizona, Tucson, AZ, EUA.

Brainerd, C. J., Reyna, V. F. \& Kneer, R. (1995). False-recognition reversal: When similarity is distinctive. Journal of Memory and Language, 34, 157185.

Brainerd, C. J., Stein, L. M. \& Reyna, V. F. (1998). On the development of conscious and unconscious memory. Developmental Psychology, 34, 342357.

Brainerd, C. J., Writght, R., Reyna, V. F. \& Mojardin, A. H. (no prelo). Conjoint recognition and phantom recollection. Journal of Experimental Psychology: Learning, Memory and Cognition.

Bransford, J. D. \& Franks, J. J. (1971). The abstraction of linguistic ideas. Cognitive Psychology, 2, 231-380.

Ceci, S. J. \& Bruck, M. (1995). Jeopardy in the courtroom. Washington, DC: American Psychological Association.

Deese, J. (1959). On the prediction of occurrence of particular verbal intrusions in immediate recall. Journal of Experimental Psychology, 58, $17-22$.

Johnson, M. K., Hashtroudi, S. \& Lindsay, S. (1993). Source monitoring. Psychological Bulletin, 114, 3-28.

Kintsch, W., Welsch, D., Schmalhofer, F. \& Zimny (1990). Sentence memory: A theoretical analysis. Journal of Memory and Language, 29, 133-159.

Loftus, E. F. (1979). Eyewitness testimony. Cambridge, MA: Harvard University Press.

Loftus, E. F. \& Hoffman, H. G. (1989). Misinformation in memory: The creation of new memories. Journal of Experimental Psychology: General, 118, 100-104.

Loftus, E. F., Miller, D. G. \& Burns, H. J. (1978). Semantic integration of verbal information into visual memory. Journal of Experimental Psychology: Human Learning and Memory, 4, 19-31.

Loftus, E. F. \& Palmer, J. C. (1974). Reconstruction of automobile destruction: An example of the interaction between language and memory. Journal of Verbal Learning and Verbal Behavior, 13, 585-589.

Maylor, E. A. \& Mo, A. (1999). Effects of study-test modality on false recognition. British Journal of Psychology, 90, 477-493.

McDermott, K. B. (1996a). The persistence of false memories in list recall. Journal of Memory and Language, 35, 212-230.

McDermott, K. B. (1996b, Novembro). Testing enhances the illusion of remembering. Trabalho apresentado no $3^{\text {th }}$ Annual Meeting of the Psychonomic Society, Chicago, IL.

McDermott, K. B. \& Roediger, H. L., III (1998). Attempting to avoid illusory memories: Robust false recognition of associates persists under conditions of explicit warnings and immediate testing. Journal of Memory and Language, 39, 508-520.

McNicol, D. (1972). A primer of signal detection theory. Londres: Allen \& Unwin.

Murdock, B. B. Jr. (1962). The serial position effect of free recall. Journal of Experimental Psychology, 64, 482-488.

Payne, D. G., Elie, C. J., Blackwell, J. M. \& Neuschatz, J. S. (1996). Memory illusions: Recalling, recognizing, and recollecting events that never occurred. Journal of Memory and Language, 35, 261-285.

Piaget, J. \& Inhelder, B. (1973). Memory and intelligence. New Your: Basic Books.

Reyna, V. F. (1995). Interference effects in memory and reasoning: A fuzzy-trace theory analysis. Em F. N. Dempster \& C. J. Brainerd (Orgs.), New perspectives on interference and inbibition in cognition (pp. 29-61). New York: Academic Press. 
Reyna, V. F. (1998). Fuzzy-trace theory and false memory. Em M. J. IntonsPeterson \& D. L. Best (Orgs.), Memory distortions and their prevention (pp. 15-27). New York: Wiley \& Sons.

Reyna, V. F. \& Brainerd, C. J. (1995). Fuzzy-trace theory: Some foundational issues. Learning and Individual Differences, 7, 145-162.

Reyna, V. F. \& Brainerd, C. J. (1998). Fuzzy-trace theory and false memory: New frontiers. Journal of Experimental Child Psychology, 71, 194-209.

Reyna, V. F. \& Kiernan, B. (1994). The development of gist versus verbatim memory in sentence recognition: Effects of lexical familiarity, semantic content, encoding instructions, and retention interval. Developmental Psychology, 30, 178-191.

Reyna, V. F. \& Lloyd, F. J. (1997). Theories of false memories in children and adults. Learning and Individual Differences, 9, 95-123.

Robinson, K. J. \& Roediger, H. L., III, (1997). Associative processes in false recall and false recognition. Psychological Science, 8, 231-237.

Roediger, H. L. III (1996). Memory illusions. Journal of Memory and Language, 35, 76-100.

Roediger, H. L., III \& McDermott, K. B. (1995). Creating false memories: remembering words not presented on lists. Journal of Experimental Psychology: Learning, Memory, and Cognition, 21, 803-814.

Roediger, H. L. III. \& McDermott, K. B. (2000). Distortions of memory. Em E. Tulving \& F. I. M. Craik (Orgs.), The Oxford Handbook of Memory (pp. 149-162). Oxford, England: Oxford University Press.

Roediger, H. L., III, McDermott, K. B. \& Robinson, K. J. (1998). The role of associative processes in creating false memories. Em M. A. Conway, S. E. Gathercole \& C. Cornoldi (Orgs.), Theories of memory II (pp. 187 245). Hove, Sussex: Psychological Press.

Russell, W. A. \& Jerkins, J. J. (1954). The Complete Minnesota Norms for Responses to 100 Words from the Kent-Rosanoff Word Association Test. Minneapolis: University of Minnesota.
Schacter, D. L., Verfaellie, M. \& Pradere, D. (1996). The neuropsychology of memory illusions: false recall and recognition in amnesic patients. Journal of Memory and Language, 35, 2319-2334.

Seamon, J. G., Luo, C. R., Schlegel S. E., Greene, S. E. \& Goldenberg, A. B. (2000). False memory for categorized pictures and words: The category associates procedure for studying memory errors in children and adults. Journal of Memory and Language, 42, 120-146.

Stadler, M. A., Roediger, H. L. \& McDermott, K. B. (1999). Norms for words that create false memories. Memory \& Cognition, 27, 494-500.

Stein, L. M. (1998). Memory falsification in children: A developmental study of spontaneous and implanted false memories. Tese de Doutorado não publicada., Department of Educational Psychology, University of Arizona. Tucson, Arizona, EUA.

Titcomb, A. L. (1996). False memories and furzy-trace theory: Misinforming gist versus verbatim memory. Tese de Doutorado não publicada. Department of Educational Psychology, University of Arizona. Tucson, Arizona, EUA.

Tsai, A., Loftus, E. \& Polage, D. (2000). Current directions in false-memory research. Em D. F. Bjorklund (Org.), False-memory creation in children and adults (pp. 31-44). Mahwah, New Jersey: Lawrence Erlbaum.

Tulving, E. (1983). Elements of episodic memory. Oxford: Oxford University Press.

Zechmeister, J. S. \& Zechmeister, E. B. (1996, Novembro). False Recall and Recognition of List Items Following Delay. Trabalho apresentado no $37^{\text {th }}$ Annual Meeting of the Psychonomic Society, Chicago, IL.

Recebido: 1/12/2000

Revisado: 26/5/2001

Aceite final: 9/6/2001

Sobre os autores:

Lilian Milnitsky Stein é Ph.D, Professora Adjunta e Coordenadora do Grupo de Pesquisa em

Processos Cognitivos do Pós-Graduação em Psicologia da Faculdade de Psicologia da Pontifícia

Universidade Católica do Rio Grande do Sul.

Giovanni Kuckartz Pergher é bolsista de Iniciação Científica - CNPq, aluno da Graduação da Faculdade de Psicologia da Pontifícia Universidade Católica do Rio Grande do Sul. 


\section{Anexo A}

\section{Lista de Palavras Associadas Apresentadas aos Participantes Material Original}

\begin{tabular}{|c|c|c|c|c|}
\hline 001. Cama & 031. Nota & 061. Mulher & 091. Nariz & 121. Quente \\
\hline 002. Descanso & 032. Som & 062. Marido & 092. Respirar & 122. Neve \\
\hline 003. Acordar & 033. Piano & 063. Tio & 093. Fungar & 123. Morno \\
\hline 004. Cansado & 034. Cantar & 064. Cavalheiro & 094. Aroma & 124. Inverno \\
\hline 005. Sonho & 035. Rádio & 065. Masculino & 095. Ouvir & 125. Gelo \\
\hline 006. Despertador & 036. Banda & 066. Pai & 096. Ver & 126. Molhado \\
\hline 007. Soneca & 037. Melodia & 067. Forte & 097. Narina & 127. Glacial \\
\hline 008. Cobertor & 038. Buzinar & 068. Amigo & 098. Odor & 128. Gélido \\
\hline 009. Cochilo & 039. Concerto & 069. Barba & 099. Olfato & 129. Calor \\
\hline 010. Sono & 040. Instrumento & 070. Pessoa & 100. Fumaça & 130. Tempo \\
\hline 011. Ronco & 041. Sinfonia & 071. Bonito & 101. Fedor & 131. Congelar \\
\hline 012. Sesta & 042. Jazz & 072. Músculo & 102. Fragrância & 132. Ar \\
\hline 013. Paz & 043. Orquestra & 073. Terno & 103. Perfume & 133. Tremor \\
\hline 014. Bocejar & 044. Arte & 074. Velho & 104. Lavanda & 134. Ártico \\
\hline 015. Sonolento & 045. Ritmo & 075. Dama & 105. Rosa & 135. Geada \\
\hline 016. Branco & 046. Azedo & 076. Teia & 106. Roubar & 136. Mesa \\
\hline 017. Escuro & 047. Bala & 077. Inseto & 107. Assaltante & 137. Sentar \\
\hline 018. Gato & 048. Açúcar & 078. Percevejo & 108. Trapaceiro & 138. Pernas \\
\hline 019. Queimado & 049. Amargo & 079. Medo & 109. Arrombador & 139. Assento \\
\hline 020. Noite & 050. Bom & 080. Voar & 110. Dinheiro & 140. Poltrona \\
\hline 021. Funeral & 051. Sabor & 081. Aracnídeo & 111. Polícia & 141. Escrivaninha \\
\hline 022. Cor & 052. Dente & 082. Rastejar & 112. Mau & 142. Reclinável \\
\hline 023. Luto & 053. Gostoso & 083. Tarântula & 113. Assaltar & 143. Sofá \\
\hline 024. Azul & 054. Mel & 084. Veneno & 114. Cadeia & 144. Madeira \\
\hline 025. Morte & 055. Refrigerante & 085. Picada & 115. Arma & 145. Estofado \\
\hline 026. Tinta & 056. Chocolate & 086. Horripilante & 116. Vilão & 146. Giratória \\
\hline 027. Fundo & 057. Coração & 087. Animal & 117. Crime & 147. Banqueta \\
\hline 028. Carvão & 058. Bolo & 088. Feio & 118. Banco & 148. Sentado \\
\hline 029. Marron & 059. Pudim & 089. Antenas & 119. Bandido & 149. Balançando \\
\hline 030. Cinza & 060. Torta & 090. Pequeno & 120. Criminoso & 150. Encosto \\
\hline
\end{tabular}


Anexo B

\section{Instruções para Aplicação dos Testes de Memória Imediato e de Memória Posterior}

\section{Rapport de Apresentação}

Boa tarde pessoal! Meu nome é Giovanni, sou estudante aqui da graduação da psicologia PUC, sou membro do Grupo de Pesquisa em Processos Cognitivos, coordenado pela professora Lilian Stein. Nosso objetivo é realizar uma pesquisa relacionada à memória e contamos com a colaboração de vocês. Os resultados desta pesquisa serão apresentados para vocês antes do final deste semestre. A participação de vocês é voluntária e anônima. Nosso trabalho terá duas etapas - uma hoje e outra semana que vem.

Vocês irão escutar uma gravação de áudio de uma lista de palavras, lidas uma de cada vez. Peço que escutem com muita atenção a cada uma delas, por que depois eu irei testar a memória de vocês para estas palavras.

Peço-lhes que não anotem nada enquanto estiverem escutando a fita, nem conversem com seus colegas. Solicito também para retirar todos objetos de cima de suas mesas.

Alguma dúvida?

Bom, podemos então começar.

\section{Instruções do Teste de Memória Imediato}

Peço-lhes que preencham na folha de respostas a idade, sexo, e turma de vocês, lembrando, mais uma vez, que o trabalho é anônimo. Portanto não incluam nenhuma informação que possa identificá-los. O que nos interessa são os dados do grupo como um todo. É importante que não deixem nenhuma questão em branco e, por favor, lembremse que o trabalho é individual. Assim sendo, não olhem para as respostas do colega ao lado e respondam de acordo com a sua própria memória.

Agora eu vou testar a memória de vocês para aquelas palavras que vocês escutaram na fita. Eu irei ler uma lista de palavras, sendo que algumas delas são exatamente iguais as da fita, e outras são palavras novas, diferentes da lista do gravador. Eu vou ler uma palavra de cada vez e vocês marcarão com um "X" na coluna do "SIM" se vocês recordarem que esta palavra é exatamente igual a palavra que vocês escutaram na fita, e marcarão com um " $\mathrm{X}$ " na coluna do "NÃO" se vocês não escutaram esta palavra no gravador.

Por exemplo, se vocês escutaram "automóvel" na lista da fita, agora eu posso falar "carro", mas vocês não devem marcar "SIM", pois, apesar das duas palavras referirem-se ao mesmo objeto, ou seja, terem o significado semelhante, elas não são a mesma palavra. Portanto, vocês devem marcam "NÃO” para carro.

Outro exemplo: Se vocês escutaram "PÊRA" na primeira lista, agora eu posso falar "MAÇÃ", aí então o que vocês marcarão? "SIM" ou "NÃO”? Entenderam? Então, marquem "SIM" somente quando a palavra for exatamente igual a da fita e marquem "NÃO” quando acharem que vocês não escutaram esta palavra anteriormente.

Lembrem-se de não deixar nenhuma resposta em branco. Certo? Então, vamos começar. Atenção para lista!

(Ao final da lista)

Obrigado pela atenção e paciência de vocês. Semana que vem vamos dar prosseguimento a este trabalho. Lembremse de guardar o número que vocês receberam.

\section{Instruções do Teste de Memória Posterior}

Boa tarde pessoal! Hoje vamos dar prosseguimento a atividade que começamos na semana passada. Cada um vai receber uma folha de resposta, e eu peço que preencham com a idade, sexo e turma de vocês 
Vale lembrar, mais uma vez, que este trabalho é voluntário e anônimo e o que nos interessa são os dados do grupo como um todo. É importante que não deixem nenhuma questão em branco e, por favor, lembrem-se que o trabalho é individual. Assim sendo, não olhem para as respostas do colega ao lado e respondam de acordo com a sua própria memória.

Agora eu vou testar a memória de vocês para aquelas palavras que vocês escutaram na fita, semana passada. Eu irei ler uma lista de palavras, sendo que algumas delas são exatamente iguais as da fita, e outras são palavras novas, diferentes da lista do gravador. Eu vou ler uma palavra de cada vez e vocês marcarão com um "X" na coluna do "SIM" se vocês recordam que esta palavra é exatamente igual a palavra que vocês escutaram na fita e marcarão com um "X" na coluna do "NÃO" se vocês não escutaram esta palavra no gravador.

Por exemplo, se vocês escutaram "montanha" na fita, agora eu posso falar "morro", mas vocês não devem marcar "SIM", pois, apesar das duas terem o mesmo sentido, elas não são a mesma palavra. Portanto, vocês devem marcam "NÃO" para morro. Então, marquem "SIM" somente quando a palavra for exatamente igual a da fita

Lembrem-se de não deixar nenhuma resposta em branco e não olhar para as respostas do colega ao lado. Certo? Então, vamos começar. Atenção para lista!

(Ao final da lista)

Obrigado pela atenção e paciência de vocês. Antes do final do semestre os resultados desta pesquisa serão apresentados para vocês. 